\title{
Theoretical Methods for Wave Propagation across Jointed Rock Masses
}

\author{
A. Perino $\cdot$ J. B. Zhu $\cdot$ J. C. Li $\cdot$ G. Barla $\cdot$ \\ J. Zhao
}

Received: 25 May 2010/ Accepted: 30 July 2010/Published online: 13 August 2010

(C) Springer-Verlag 2010

\begin{abstract}
Different methods are presently available for the analysis of wave propagation across jointed rock masses with the consideration of multiple wave reflections between joints. These methods can be divided into two categories. One is based on the displacement discontinuity model for representing rock joints, where the displacements across a joint are discontinuous and the tractions are continuous, and the other is the equivalent medium method. For the first category, there are three methods, i.e., method of characteristics (MC), scattering matrix method (SMM) and virtual wave source method (VWS). MC solves the equation of motion by using the theory of characteristic curves. SMM is based on the definition of the scattering matrix in which the reflection and transmission coefficients of a set of joints are stored. VWS method replaces the joints in the rock mass with a virtual concept. For the second category, equivalent medium model treats the problem in the frame of continuum mechanics and simplifies it from an explicit wave propagation equation. The objective of this paper is to review and compare these theoretical methods. The comparison shows that the four solutions agree very well with each other. Some additional
\end{abstract}

\footnotetext{
A. Perino $\cdot$ G. Barla

Department of Structural and Geotechnical Engineering, Politecnico di Torino, Turin, Italy

J. B. Zhu · J. C. Li · J. Zhao (ه)

Laboratory for Rock Mechanics (LMR),

Ecole Polytechnique Fédérale de Lausanne (EPFL),

Lausanne, Switzerland

e-mail: jian.zhao@epfl.ch

J. C. $\mathrm{Li}$

State Key Laboratory of Geomechanics and Geotechnical Engineering, Institute of Rock and Soil Mechanics, Chinese Academy of Sciences, Wuhan, China
}

considerations about the advantages and disadvantages of these methods are also given in the paper.

Keywords Wave propagation - Rock joints · Displacement discontinuity method $\cdot$ Equivalent medium model

\section{Introduction}

The rock mass is characterized by the existence of joints, which are discontinuities in parallel form. The effects of the joints on wave propagation are essential for the geophysical and dynamic behavior of the rock mass. Therefore, the prediction of wave attenuation across jointed rock masses is very important in solving problems in geophysics, seismic investigations, rock dynamics, rock protective engineering and earthquake engineering.

The theoretical methods for studying the influence of joints on the elastic wave propagation are essentially based on two different models, namely, displacement discontinuity model (DDM) and equivalent medium model (EMM). The DDM was originally developed by Mindlin (1960) and applied to seismic wave propagation by Schoenberg (1980). The basic assumption of this method is that the particle displacements of a seismic wave as it propagates through a joint are discontinuous, while the stresses remain continuous. Myer et al. (1990) and PyrakNolte et al. (1990a) extended the method to fluid saturated joints (Kelvin and Maxwell model). Pyrak-Nolte (1988) and Cook (1992) used the displacement discontinuity method (DDM) for studying the influence of a set of parallel joints on the transmission of seismic waves. Other applications of this method are given by Cai and Zhao (2000), Zhao J et al. (2006) and Zhao XB et al. (2006a, b), 
who used the method of characteristics (MC) for solving wave propagation across a set of parallel joints, where multiple wave reflections were considered. Besides linearly deformational joints, joints with nonlinear (Zhao and Cai 2001; Zhao J et al. 2006) and Coulomb slip behavior (Zhao $\mathrm{XB}$ et al. 2006a) were also studied with MC. The influence of frictional interfaces was analyzed by Miller (1978).

Some studies of elastic wave propagation in fractured media involved the use of EMM, where effective elastic moduli were calculated and used to represent the fractured medium (e.g., Hudson 1981; Frazer 1990; Coates and Schoenberg 1995; Slawinski 1999). The negative aspects of this approach (White 1983; Schoenberg and Muir 1989; Pyrak-Nolte et al. 1990b; Zhao J et al. 2006; Zhao XB et al. $2006 \mathrm{a}, \mathrm{b})$ are due to the simplification of the discontinuous rock mass to an equivalent medium. This approach cannot work well in representing media where the fractures are relatively large and sparsely spaced (with spacing of the order of, or larger than, a seismic wavelength) (PyrakNolte et al. 1990b). Recently, Li et al. (2010) proposed an equivalent viscoelastic medium model, which was composed of a viscoelastic medium model and the concept of virtual wave source (VWS). By using continuum mechanics, the equivalent viscoelastic medium model analytically studied wave propagation across the rock mass by solving an explicit wave propagation equation, so as to simplify the problem.

The purpose of this paper is to present four different methods for simulating wave propagation across multiple rock joints, where multiple wave reflections are taken into account. From the four methods, the transmitted waves across a rock mass with one joint set are compared. The advantages and disadvantages of these presented methods are then discussed.

\section{Theoretical Methods for Wave Propagation across Jointed Rock Masses}

When a stress wave propagates across a joint set, multiple wave reflections among the joints have a great effect on the transmitted wave. In fact, the transmitted wave can be seen as the superposition of waves arriving at different time. In this section, four different theoretical methods for wave propagation across one joint set are introduced, where multiple wave reflections are taken into account.

\subsection{Displacement Discontinuity Method}

The DDM is a widely used method to model the fracture in a medium. This method models the boundary (or fractures and joints in rock mass) between two elastic, homogeneous and isotropic half-spaces as being in non-welded contact.
The boundary conditions along the fracture are the discontinuity of displacements and the continuity of tractions. The small vector difference in the displacement is assumed to depend linearly on the traction vector. This interface condition, called a "linear slip condition", replaces the condition of continuous displacement (Schoenberg, 1980).

The linear discontinuity models for dry fractures are valid, provided that the magnitude of seismic stress is insufficient to mobilize the nonlinear asperity deformation and frictional slip of the fracture (Yi et al. 1997). This situation is typical in engineering practice such as ultrasonic detection and most seismic investigations in fractured rock masses, where the wave amplitudes are relatively small.

The fracture or rock joints considered with DDM are planar, large in extent and small in thickness compared to the wavelength.

\subsubsection{Method of Characteristics}

MC has been widely used to study one-dimensional wave propagation in continuous media (Ewing et al. 1957; Courant and Hilbert 1962; Bedford and Drumheller 1994). Based on one-dimensional wave equation, relations between particle velocity and stress along right- and leftrunning characteristics can be built. As shown in Fig. 1, the quantity $(Z v+\sigma)$ is a constant along any straight line with slope $1 / \alpha$ (right-running characteristic) in the $x-t$ plane, where $\sigma$ is the normal stress, $v$ is the wave velocity, $Z=\rho \alpha$ is the impedance of the material, $\rho$ is the rock density, and $\alpha$ the wave velocity. Similarly, the quantity $(Z v-\sigma)$ is a constant along any straight line with slope of $-1 / \alpha$ (left-running characteristic) in the $x-t$ plane. It is noted that the normal stress is defined to be positive for compressive stress, and negative for tensile stress. The definition is consistent with that commonly used in rock mechanics.

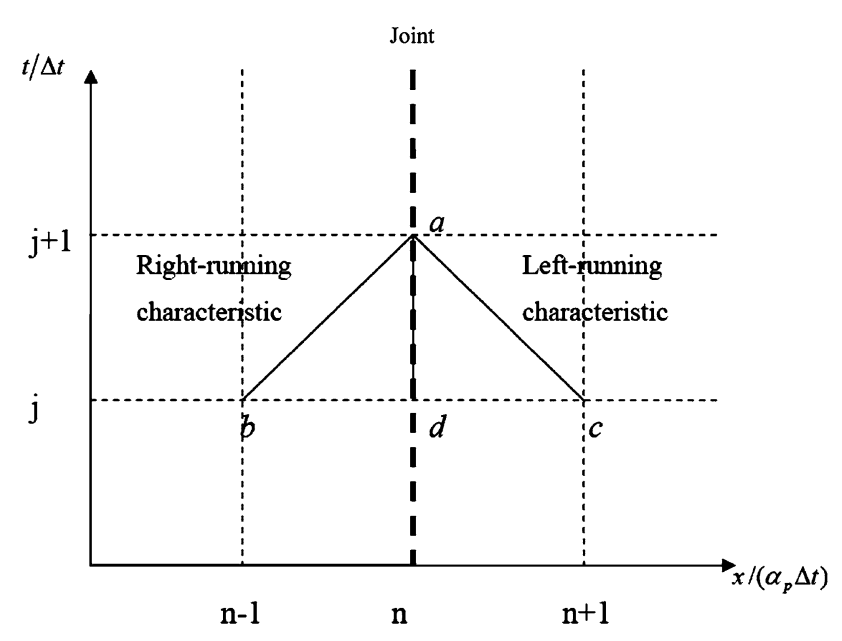

Fig. 1 Characteristics in the nondimensional $x-t$ plane 
MC can also be used to study wave propagation across discontinuous jointed rock masses (Cai and Zhao 2000; Zhao J et al. 2006; Zhao XB et al. 2006a, b). When a wave propagates in jointed rock masses, multiple reflections occur between joints. Actually, the transmitted wave across parallel joints can be treated as wave superposition of differently arriving transmitted waves caused by multiple reflections. In the $x-t$ plane, new variables, nondimensional distance $(n)$ and nondimensional time $(j)$, are imported:

$j=t / \Delta t$,

$n=x /(\alpha \Delta t)$,

where $\Delta t$ is the time interval.

It is assumed that a finite number of interfaces are located at integral values of nondimensional distance in a half space with its left boundary at $n=0$, the first interface at $n=1$, the second interface at $n=2$, and the last interface at $n=l(l$ is an integer). The interface could be a joint or a welded interface, which can be treated as a joint with infinite joint specific stiffness. Figure 1 shows conjunction points of right- and leftrunning characteristics at integral values of nondimensional distance and nondimensional time. Particle velocities and normal stresses are evaluated at these points. However, this does not mean that solutions can be obtained only at the interface positions. If the field between two adjacent interfaces is further divided into certain number of layers, solutions can be obtained at these layers' boundaries, which are treated as joints with infinite joint specific stiffness.

In the analysis, it is assumed that joints and elastic media on both sides of the joints have identical properties. Along the right-running characteristic $a b$ and the left-running characteristic $a c$ shown in Fig. 1, two relations between particle velocities and normal stresses at points $a, b$ and $c$ are built:

$$
\begin{aligned}
Z v^{-}(n, j+1)+\sigma^{-}(n, j+1)= & Z v^{+}(n-1, j) \\
& +\sigma^{+}(n-1, j), \\
Z v^{+}(n, j+1)-\sigma^{+}(n, j+1)= & Z v^{-}(n+1, j) \\
& -\sigma^{-}(n+1, j),
\end{aligned}
$$

where $v^{-}(n, j+1)$ and $v^{+}(n, j+1)$ are particle velocities at time $j+1$ before and after the joint at distance $n$, and $\sigma^{-}(n, j+1)$ and $\sigma^{+}(n, j+1)$ are normal stresses at time $j+1$ before and after the joint at distance $n$.

Based on the DDM, the stresses at the joint are continuous, while the displacements are discontinuous. Therefore, the response at point $a$ can be derived:

$$
\begin{aligned}
& \sigma^{-}(n, j+1)=\sigma^{+}(n, j+1)=\sigma(n, j+1), \\
& u^{-}(n, j+1)-u^{+}(n, j+1)=\sigma(n, j+1) / k_{n},
\end{aligned}
$$

where $k_{n}$ is the normal joint specific stiffness, and $u^{-}(n, j+1)$ and $u^{+}(n, j+1)$ are displacements at time $j+1$ before and after the joint at distance $n$.
With Eq. 5, the addition of Eqs. 3 and 4 is

$$
\begin{aligned}
Z v^{-}(n, j+1)+Z v^{+}(n, j+1)= & Z v^{+}(n-1, j) \\
& +\sigma^{+}(n-1, j) \\
& +Z v^{-}(n+1, j) \\
& -\sigma^{-}(n+1, j)
\end{aligned}
$$

The differentiation of Eq. 6 with respect to $t$ is

$v^{-}(n, j+1)-v^{+}(n, j+1)=\frac{1}{k_{n}} \frac{\partial \sigma(n, j+1)}{\partial t}$

If $\Delta t$ is small enough, Eq. 8 can be expressed as

$v^{-}(n, j+1)-v^{+}(n, j+1)=\frac{\sigma(n, j+1)-\sigma(n, j)}{k_{n} \Delta t}$

Equation 9 can be rewritten as

$\sigma(n, j+1)=\sigma(n, j)+k_{n} \Delta t\left[v^{-}(n, j+1)-v^{+}(n, j+1)\right]$

Substituting Eq. 10 into Eq. 3 gives

$$
\begin{aligned}
& \left(k_{n} \Delta t+Z\right) v^{-}(n, j+1)-k_{n} \Delta t v^{+}(n, j+1)+\sigma(n, j) \\
& \quad=Z v^{+}(n-1, j)+\sigma(n-1, j)
\end{aligned}
$$

Equations 7 and 11 form a linear equation group with respect to particle velocities at point $a$ before and after the joint. After it is solved, expressions of particle velocities at point $a$ are obtained:

$$
\begin{aligned}
v^{-}(n, j+1)= & \left(Z v^{+}(n-1, j)+\sigma(n-1, j)-\sigma(n, j) .\right. \\
& +\frac{k_{n} \Delta t}{Z}\left(Z v^{-}(n+1, j)+Z v^{+}(n-1, j)\right. \\
& +\sigma(n-1, j)-\sigma(n+1, j))) /\left(2 k_{n} \Delta t+Z\right)
\end{aligned}
$$

$$
\begin{aligned}
v^{+}(n, j+1)= & \left(\left(Z v^{+}(n-1, j)-\sigma(n+1, j)\right.\right. \\
& \left.+\sigma(n-1, j)+Z v^{-}(n+1, j)\right) \frac{k_{n} \Delta t+Z}{Z} \\
& -Z v^{+}(n-1, j)+\sigma(n, j) \\
& -\sigma(n-1, j)) /\left(2 k_{n} \Delta t+Z\right)
\end{aligned}
$$

By substituting Eqs. 12 and 13 into Eq. 10, the expression of normal stress is obtained:

$$
\begin{aligned}
\sigma(n, j+1)= & \sigma(n, j)+\frac{k_{n} \Delta t}{2 k_{n} \Delta t+Z}\left(Z v^{+}(n-1, j)+\sigma(n-1, j) .\right. \\
& \left.-Z v^{-}(n+1, j)+\sigma(n+1, j)-2 \sigma(n, j)\right)
\end{aligned}
$$

Equations 12-14 show that the responses at point $a$ are determined by those at points $b, c$ and $d$. Meanwhile, it indicates that responses at time $j+1$ can be calculated from those at time $j$. With input velocity of $v(0, j)$ and initial conditions of $v^{+}(n, 0), v^{-}(n, 0)$ and $\sigma(n, 0)$, Eqs. 11-13 are applied to determine particle velocities and stress at any point through an iterative computation. 


\subsubsection{Scattering Matrix Method}

The scattering phenomenon that takes place when an elastic wave impinges on a discontinuity is conveniently described by a scattering matrix (S) (Aki and Richards 2002). In the case of a planar interface between media with different elastic properties or in the case of a planar joint in a rock mass, incident, reflected and transmitted plane waves have the same transverse wave vector. The respective amplitudes are related by a $2 \times 2$ block matrix

$\mathbf{S}=\left(\begin{array}{ll}\mathbf{S}_{11} & \mathbf{S}_{12} \\ \mathbf{S}_{21} & \mathbf{S}_{22}\end{array}\right)$

where $\mathbf{S}_{i i}$ have the meaning of reflection coefficients at the two sides of the interface and $\mathbf{S}_{i j}$ are transmission coefficients. Since elastic waves have three possible polarization states $(\mathrm{P}, \mathrm{SV}, \mathrm{SH})$, the submatrices have the size of $3 \times 3$.

When more parallel discontinuities are presented, the scattering matrices of each one are combined according to a standard algorithm to describe the behavior of the complete structure, with the consideration of all multiple reflected waves.

The method is borrowed from the study of electromagnetic waves propagation and the theory of transmission lines such as coaxial cables, optical fibers, strip-lines, etc. (Collin 1992).

It is well known that the propagation of plane elastic waves in a stratified medium can be conveniently analyzed by a transmission line formalism. Let $z$ be the stratification axis. The variables used to describe the elastic field are the velocity $\mathbf{v}$ and the $-\mathbf{T} \cdot z$ traction on a surface orthogonal to $z$.

The $z$ dependent part of a generic plane wave field, with any incidence angle, can be written as:

$\left(\begin{array}{c}\mathbf{v} \\ -\mathbf{T} \cdot z\end{array}\right)=\mathbf{M}\left(\begin{array}{l}\mathbf{c}^{+} \\ \mathbf{c}^{-}\end{array}\right)$

where the $\mathbf{v}$ portion is analogous to an equivalent current and the $-\mathbf{T} \cdot z$ one to an equivalent voltage.

The columns of the $6 \times 6$ modal matrix contain the vector parts of the forward and backward $\mathrm{SH}, \mathrm{SV}, \mathrm{P}$ waves. At a planar interface between different media "1" and " 2 " (Fig. 2), the state vector is continuous; hence from

$\mathbf{M}_{1}\left(\begin{array}{l}\mathbf{c}_{1}^{+} \\ \mathbf{c}_{1}^{-}\end{array}\right)=\mathbf{M}_{2}\left(\begin{array}{l}\mathbf{c}_{2}^{+} \\ \mathbf{c}_{2}^{-}\end{array}\right)$

we obtain the transmission matrix that relates the wave amplitudes at the two sides

$\mathbf{A}=\mathbf{M}_{1}^{-1} \mathbf{M}_{\mathbf{2}} \quad\left(\begin{array}{l}\mathbf{c}_{1}^{+} \\ \mathbf{c}_{1}^{-}\end{array}\right)=\mathbf{A}\left(\begin{array}{l}\mathbf{c}_{2}^{+} \\ \mathbf{c}_{2}^{-}\end{array}\right)$

By simplifying algebraic manipulations, we get the corresponding scattering matrix

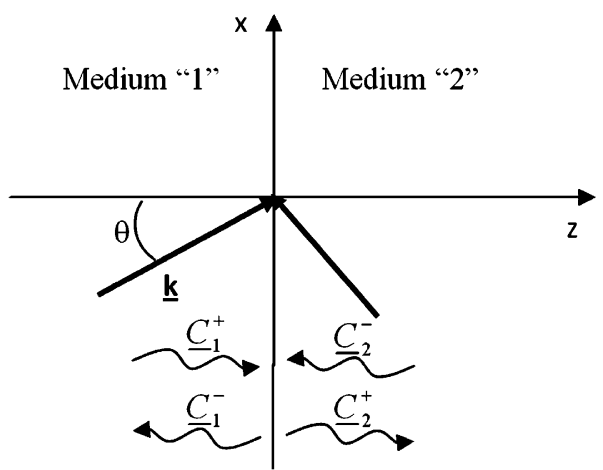

Fig. 2 Wave propagation through an interface

$\left(\begin{array}{l}\mathbf{c}_{1}^{-} \\ \mathbf{c}_{2}^{+}\end{array}\right)=\left(\begin{array}{ll}\mathbf{S}_{11} & \mathbf{S}_{12} \\ \mathbf{S}_{21} & \mathbf{S}_{22}\end{array}\right)\left(\begin{array}{l}\mathbf{c}_{1}^{+} \\ \mathbf{c}_{2}^{-}\end{array}\right)$

Note that $\mathbf{c}_{1}^{+}$and $\mathbf{c}_{2}^{-}$are the amplitudes of the waves incident on the discontinuity, whereas $\mathbf{c}_{1}^{-}$and $\mathbf{c}_{2}^{+}$are the amplitudes of the scattered waves (reflected and transmitted). For generality, there is also a wave from the right side (Fig. 2), but with the same value of the transverse wave vector.

As an example, with the ordering of the modal matrix as above, the element 2,3 of block $\mathbf{S}_{11}$ is the reflection coefficient for an incident pressure wave into an SV reflected wave. Since SH waves are uncoupled from the other two, it is possible to decompose the general scattering matrix into a $2 \times 2$ matrix, obtained by a scalar analysis $(\mathrm{SH})$, and a $4 \times 4$ matrix, for $\mathrm{SV}$ and $\mathrm{P}$ waves.

Another type of discontinuity that we have considered is the joint between homogeneous media, described by a displacement discontinuity. The relevant equivalent circuit is a matrix admittance $\mathbf{Y}$, connected in parallel on the transmission line representing wave propagation in the $z$ direction. The transmission matrix of this discontinuity is

$\mathbf{A}=\mathbf{M}_{1}^{-1}\left(\begin{array}{ll}1 & \mathbf{Y} \\ \mathbf{0} & 1\end{array}\right) \mathbf{M}_{\mathbf{2}}$

from which the scattering matrix is readily obtained.

For example, the coefficients for incident $\mathrm{SH}$ wave are as follows:

$R_{\mathrm{SH}}(\omega, \theta)=S_{11}=\frac{Z_{s} Y}{2+Z_{s} Y}$

$T_{\mathrm{SH}}(\omega, \theta)=S_{21}=\frac{2}{2+Z_{s} Y}$

where $Z_{s}=\rho V_{\mathrm{s}} \cos \theta$ is the impedance of the medium, $Y$ is the fracture admittance and depends on the type of discontinuity (linear elastic or with infilling material) and therefore on the stiffness and/or the specific viscosity and angular frequency $\omega$. 
It is noted that the previous equations hold true for a plane SH wave, which impinges with any oblique angle $\theta$ and is characterized by a given angular frequency $\omega$. Similar expressions can be obtained for SV or P waves normal to the discontinuity. For different angles, these waves are coupled. They are similar to the equations previously given by Myer et al. (1990).

To extend the method to the case of $N$ parallel discontinuities, one may compute the scattering matrix for each discontinuity. Then, by using a "chain rule" procedure, the global scattering matrix is defined. This is a combination of the components of the scattering matrix for each discontinuity and represents the effect on elastic wave propagation due to the $N$ discontinuities. The global scattering matrix contains the global transmission and reflection coefficients of the set of parallel discontinuities considered.

Damping can be implemented by using a viscoelastic formulation, which allows one to obtain the complex shear modulus as a function of the damping ratio of the material. In the analyses discussed in the following, material damping will be neglected so as to study the influence due to the discontinuities only. It is noted that different discontinuity types can be considered, such as linear elastic joint and filled joint (viscoelastic models).

\subsubsection{Virtual Wave Source}

Li et al. (2010) introduced a new concept of virtual wave source (VWS) with a viscoelastic medium model to establish an equivalent viscoelastic model, which can describe the dynamic properties of the rock mass by using a continuous medium model and derive an explicit expression for the wave propagation equation. Here, the VWS concept is different and extended; that is, VWS represents the mechanical properties of rock joints in a rock mass.

The VWS exists at the joint position and produces new waves when the incident wave approaches the joint. The transmitted wave across the rock mass can be treated as the superposition of different transmitted waves created by the VWS. Combined with the DDM, VWS can also be used to study wave propagation across a discontinuous rock mass, where the discreteness of the joints can be taken into account.

If an incident harmonic P-wave with the form of

$v_{I}=I \exp (i \omega t)$

transmits across a joint, the reflection and transmission coefficients across it can be written as (Pyrak-Nolte et al. 1990a)

$R_{1}=\frac{i}{-i+2 k_{n} /(Z \omega)}$
$T_{1}=\frac{2 k_{n} /(Z \omega)}{-i+2 k_{n} /(Z \omega)}$

where $I$ is the amplitude of the incident wave, $k_{n}$ is the normal joint specific stiffness, $Z$ is the wave impedance, and $\omega$ is the angular frequency. Equations 24 and 25 show that reflection and transmission coefficients are dependent on a combined parameter $K_{n}=k_{n} /(\mathrm{Z} \omega)$, which is named as normalized normal joint stiffness.

To obtain the theoretical solution of the transient wave transmitting across a joint set, the incident transient wave $\left(v_{I}\right)$ is first transformed into frequency domain by FFT (fast Fourier transform). In the frequency domain, the incident transient wave can be transformed as the sum of a series of harmonic waves with different amplitudes and frequencies:

$v_{I}=\sum_{i=-\infty}^{\infty} v_{I i}=\sum_{i=-\infty}^{\infty} A_{i} e^{i \omega_{i} t}$

where $v_{I i}$ is one harmonic wave, and $A_{i}$ and $\omega_{i}$ are the amplitude and angular frequency of the harmonic wave $v_{I i}$.

When the rock is characterized by the presence of two or more joints, the transmitted wave can be treated as wave superposition of transmitted waves arriving at different times. To take into account wave superposition, the method of VWS is used. VWS exists at each joint position, represents the mechanical properties of the joint and produces two new waves with opposite directions when an incident wave impinges the VWS.

The concept of VWS can be re-explained as there are one reflected wave and one transmitted wave created from VWS, when the wave arrives at the position of VWS. The reflected and transmitted waves from one joint can be obtained by Eqs. 24 and 25 when the incident harmonic wave is known.

Figure 3 illustrates the scheme of wave propagation across a jointed rock mass with VWS. The transmitted harmonic wave across one joint set is the result of the wave superposition of different transmitted waves created by the VWS:

$v_{T i}=\sum_{j=1}^{\infty} v_{T i j}$

where $v_{T i}$ is the transmitted wave for the incident harmonic wave $v_{I i}$, and $v_{T i j}$ is the transmitted wave arriving at a different time, as shown in Fig. 3. Then, an inverse transform for these transmitted harmonic waves is conducted to get the transmitted transient wave $\left(v_{T}\right)$ by IFFT (inverse fast Fourier transform), which can transform one series of harmonic waves into a transient wave:

$v_{T}=\sum_{i=-\infty}^{\infty} v_{T i}$ 


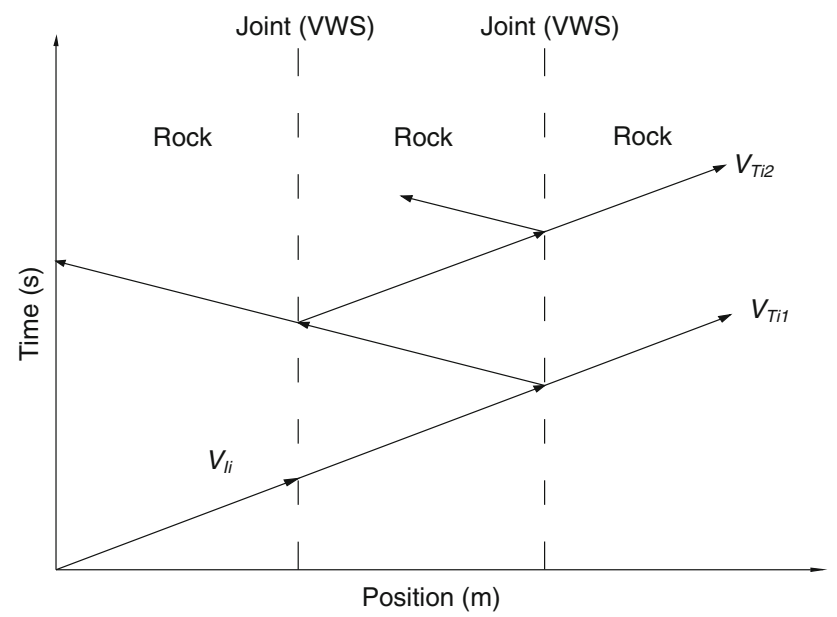

Fig. 3 Scheme of jointed rock mass with VWS (2 joints)

\subsection{Equivalent Medium Model}

The equivalent medium model (EMM) (Li et al. 2010) is a continuous medium model, which describes the dynamic property of the discontinuous rock mass. This model consists of a viscoelastic medium model and the concept of virtual wave source (VWS). The viscoelastic medium model is an auxiliary spring placed in series with the Voigt model (Fig. 4), which can display both the attenuation and the frequency dependence of the transmitted wave. The concept of VWS is to consider the effect of discreteness of a rock mass on wave propagation, i.e., the wave reflections between two joints.

As for the longitudinal motion equation for onedimensional problems, the equivalent model of the auxiliary spring placed in series with the Voigt model can be mathematically expressed as

$\rho \eta_{v} \frac{\partial^{2} v}{\partial t^{2}}+\rho\left(E_{a}+E_{v}\right) \frac{\partial v}{\partial t}-\eta_{v} E_{a} \frac{\partial^{2} v}{\partial x^{2}}-E_{v} E_{a} \int \frac{\partial^{2} v}{\partial x^{2}} \mathrm{~d} t=0$

where $v$ is the particle velocity and $E_{a}$ is the Young's modulus of the intact rock, while $E_{v}$ represents the stiffness contributed by the joints; $\eta_{v}$ is the viscosity, $\rho$ is the density

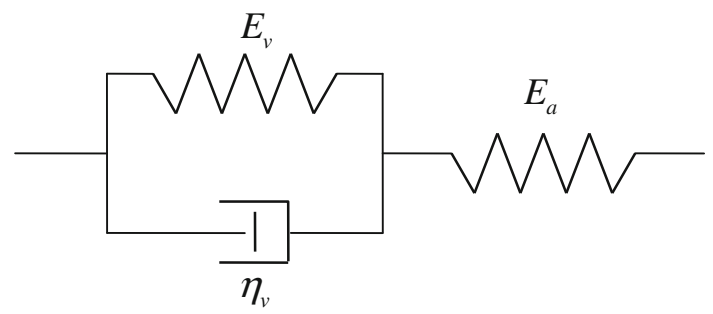

Fig. 4 Equivalent mechanical model of an auxiliary spring in series with the Voigt model of the medium, and $t$ is time. We define $\tau=\eta_{v} / E_{v}$ as the time of retardation of the Voigt element and

$\frac{1}{E_{c}}=\frac{1}{E_{a}}+\frac{1}{E_{v}}$

$E_{v}$ and $v_{v}$ need to be determined by comparing the transmitted wave through the equivalent medium with the existing solutions of a discontinuous rock mass. $E_{v}$ is obtained for each joint section (including one joint and rock material having thickness equal to the joint spacing). Therefore, joint spacing is involved in the determination of $E_{v}$, while the joint thickness is not, since the joint is very thin and the thickness is considered to be zero.

The solution for Eq. 29 has the following form

$v=A \exp (\beta x) \exp [i(-\omega t+\alpha x)]$

where $A$ is the amplitude of the incident velocity wave, $\omega=2 \pi f$ and $f$ is the frequency of the wave, and

$$
\left\{\begin{array}{l}
\alpha=\left\{\frac{\rho \omega^{2}}{2 E_{c} E_{a}}\left[\left(\frac{E_{a}^{2}+E_{c}^{2} \omega^{2} \tau^{2}}{1+\omega^{2} \tau^{2}}\right)^{1 / 2}+\frac{E_{a}+E_{c} \omega^{2} \tau^{2}}{1+\omega^{2} \tau^{2}}\right]\right\}^{\frac{1}{2}} \\
\beta=-\left\{\frac{\rho \omega^{2}}{2 E_{c} E_{a}}\left[\left(\frac{E_{a}^{2}+E_{c}^{2} \omega^{2} \tau^{2}}{1+\omega^{2} \tau^{2}}\right)^{1 / 2}-\frac{E_{a}+E_{c} \omega^{2} \tau^{2}}{1+\omega^{2} \tau^{2}}\right]\right\}^{\frac{1}{2}}
\end{array}\right.
$$

$\alpha$ gives the phase shift per unit length and the minus sign of $\beta$ indicates the wave attenuation.

Across the joint set, the final transmitted wave is the superposition of two parts. The first one is from the direct transmission of the initial incident wave and the other one is from the multiple reflections among the joints. Although the frequency dependence and wave attenuation have been shown in Eqs. 31 and 32, the effect of the discreteness of joints on wave propagation in the viscoelastic solid still cannot be reflected in the two equations.

To solve this problem, the concept of virtual wave source (VWS) is proposed in the equivalent medium model. VWS exists at each joint surface and produces a new wave (in the opposite direction of the incident wave) at each time when an incident wave propagates across the VWS. The distance between two adjacent VWSs is equal to the joint spacing $S$. The equivalent length of the medium is defined as the product of joint number $N$ and the joint spacing $S$, i.e., $N S$. Figure 5 shows a rock mass with three parallel joints and the corresponding equivalent medium with and without VWS, where the equivalent length is $3 S$. The concept of VWS can be interpreted as: a reflected wave is created from the virtual wave source when either a positive or a negative wave arrives at the VWS.

Assume that there is an incident $\mathrm{P}$-wave

$v_{I}(t, 0)=A \exp (-i \omega t)$ 
(a)

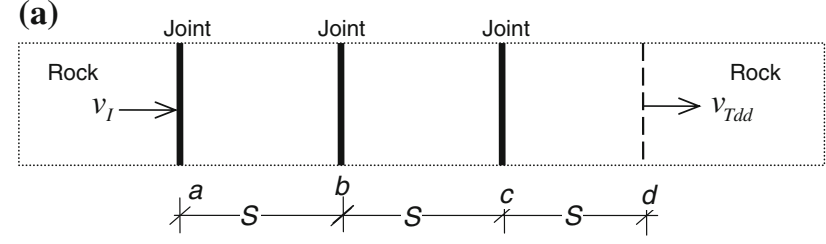

(b)
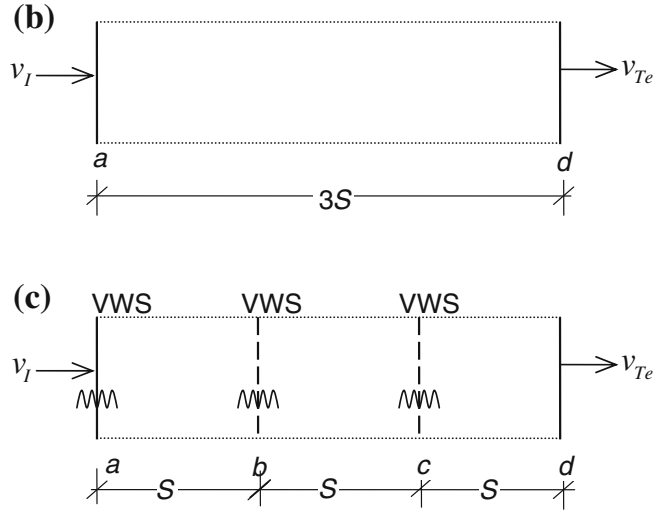

Fig. 5 Scheme of jointed rock mass and equivalent medium. a Jointed rock mass, b equivalent medium model without virtual wave source (VWS), and c equivalent medium model with virtual wave source (VWS)

from the left side $a$ of the equivalent medium in Fig. 5. According to Eq. 30, along the direction of the incident wave, the particle velocity at point $b$ is

$v_{e}(t, S)=A \exp (\beta S) \exp [i(-\omega t+\alpha S)]$

where the phase shift of $v_{e}(t, S)$ and $v_{I}(t, 0)$ is $\alpha S$. According to the energy conservation of the simple harmonic waves [Cook 1992], the amplitude of the reflected wave at the interface $b$ is $A\left\{1-[\exp (\beta S)]^{2}\right\}^{1 / 2}$, if the interface $b$ is a discontinuous boundary. From the Kramer-Kronig relation (a statement of causality), any changes in the amplitude of a wave must be accompanied by a change in phase. Since the phase shift between the reflected and transmitted waves is $\pi / 2$ [Pyrak-Nolte et al. 1990a; Cook 1992], the reflected wave at $b$ can be expressed as

$v_{e}^{\prime}(t, S)=A \sqrt{1-[\exp (\beta S)]^{2}} \exp [i(-\omega t+\alpha S-\pi / 2)]$

where $v_{e}^{\prime}(t, S)$ is regarded as the wave produced from the VWS at $b$. Then, $v_{e}(t, S)$ and the created wave $v_{e}^{\prime}(t, S)$ propagate along two opposite directions as new incident waves to the adjacent interfaces $c$ and $a$, where new waves are repeatedly created and propagate to their adjacent interfaces. The transmitted wave at the right side $d$ of the equivalent medium is a wave superposition of $v_{e}(t, 3 S)$ arriving at different times, which is the summation of multiple waves created from the three VWSs and the transmitted wave from the incident wave $v_{I}(t, 0)$ propagating across the viscoelastic medium.

\section{Comparison of Results and Discussion}

\subsection{Comparison of Results}

To compare the four different methods described above, a one-dimensional P-wave propagation analysis through a rock mass with parallel joints is performed. The joints considered in the analysis are planar, large in extent and small in thickness compared to the wavelength. The material damping is not considered because we only study the effects of joints on wave propagation. The model is the same for all the methods considered (see Fig. 5a). A normally incident half-cycle sinusoidal $\mathrm{P}$-wave is assumed to be applied at the boundary of the model with a frequency of $f_{0}=100 \mathrm{~Hz}$ and unit amplitude (Fig. 6). Hence, the incident wave is applied at the boundary " $a$ " while the transmitted wave is recorded at the boundary "d" (Fig. 5a). The mechanical properties of the rock mass are listed in Table 1.

Transmitted waveforms across $1,2,3$ and 4 parallel joints with joint spacing $S=0.1 \lambda\left(\lambda=2 \pi C / \omega_{0}\right)$ and normalized joint stiffness $K_{n}=0.36$ are plotted in Fig. 7. The transmission coefficient is defined as the ratio of the amplitude of the transmitted wave to that of the incident wave. The transmitted wave is shown to decrease in amplitude with increasing number of joints. Therefore, the incident wave is attenuated from jointed rock mass, and this attenuation increases with the number of joints. Each joint causes a time delay of the transmitted wave. This time delay increases with the joint number and results in a phase

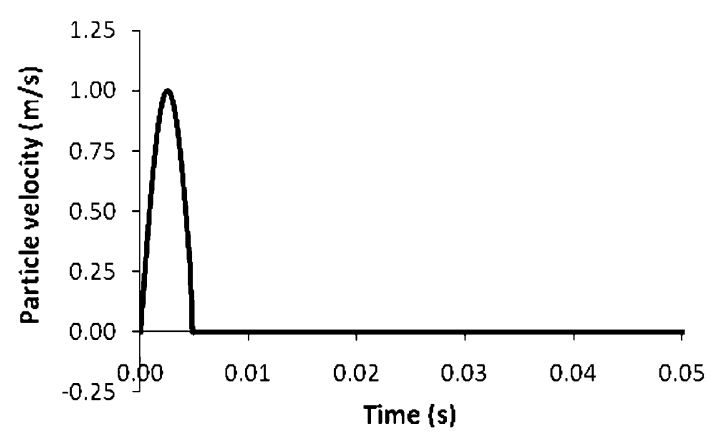

Fig. 6 Incident wave (half-cycle sinusoidal wave)

Table 1 Mechanical properties of the rock mass

\begin{tabular}{ll}
\hline Density & $2,650 \mathrm{~kg} / \mathrm{m}^{3}$ \\
Velocity of the propagating P-wave & $5,830 \mathrm{~m} / \mathrm{s}$ \\
Joint normal stiffness & $3.5 \mathrm{GPa} / \mathrm{m}$ \\
\hline
\end{tabular}


shift. Hence, the phase shift $\vartheta$ for a compressional wave normally incident upon a joint (Schoenberg, 1980) is a function of the normalized joint normal stiffness $K_{n}$ and $\vartheta=\arctan \left(1 / 2 K_{n}\right)$. Obviously, there is also the other part of this time delay that is caused by the medium between joints.

Moreover, it is clear from Fig. 7 that the transmitted waveforms obtained with all four methods are almost coincident. We can only note negligible differences for later arriving transmitted waves.

Wave propagation across a single fracture is shown in Fig. 8a. By showing the trend of the transmission coefficient $\left|T_{1}\right|$ versus the normalized joint normal stiffness $K_{n}$, it is found that the four methods agree well with each other. In Fig. 8a, we can see that if the value of $K_{n}$ approaches infinity, $\left|T_{1}\right|$ approaches 1 . While $K_{n}$ approaches $0,\left|T_{1}\right|$ approaches 0 . These two limit conditions correspond, respectively, to the case of a welded and free interface. Between these two extreme conditions, $\left|T_{1}\right|$ increases with increasing $K_{n}$.

Figure 8b shows the transmission coefficient $\left|T_{2}\right|$ evaluated for a model with two parallel joints by varying the non-dimensional ratio $(\xi)$ of joint spacing to wavelength for different values of $K_{n}(0.3,1)$. The following observations are made:

1. The four methods agree well with each other.

2. $\left|T_{2}\right|$ increases with increasing $K_{n}$.
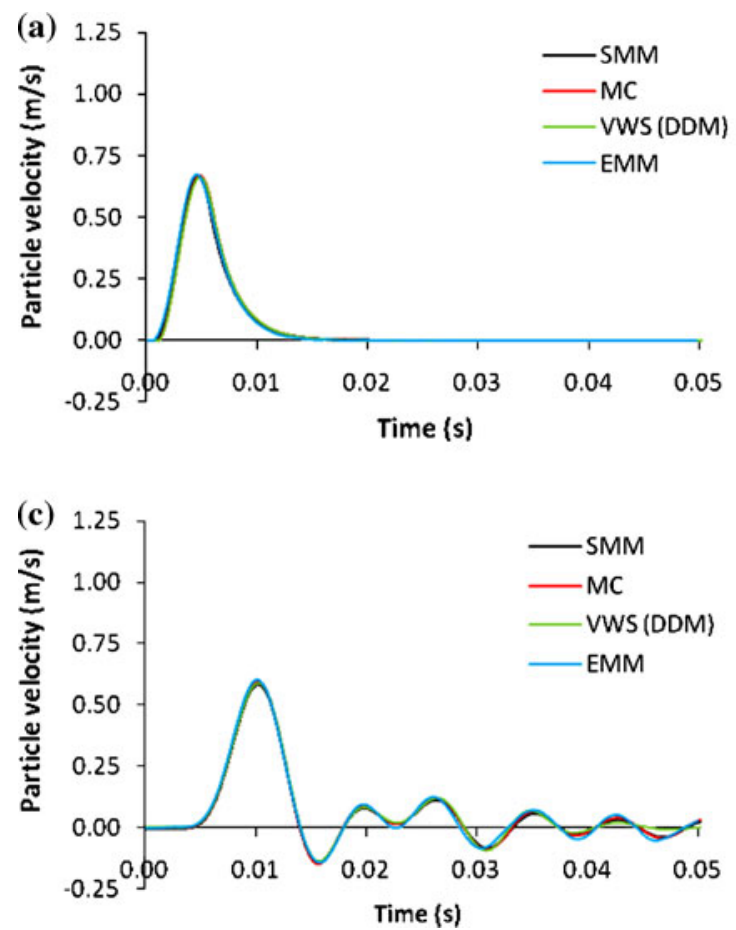

3. When $\xi>0.19$ for $K_{n}=1$ and $\xi>0.26$ for $K_{n}=0.3$, $\left|T_{2}\right|$ is constant.

4. For smaller $\xi$ values, $\left|T_{2}\right|$ reaches a maximum value. The trends of $\left|T_{2}\right|$ versus $\xi$ are essentially the same for the two values of $K_{n}$ analyzed.

5. $\left|T_{2}\right|$ increases with $K_{n}$.

Figure 8c shows the magnitude of transmission coefficients across $\mathrm{N}$ (2 and 6) joints as a function of $\xi$ with $K_{n}=0.36$. We can note that:

1. The four methods agree well. There is only a small gap between EMM and other DDMs between $\xi=0.31$ and $\xi=0.57$.

2. $\left|T_{N}\right|$ decreases with increasing $N$.

3. When $\xi>0.24$ for $N=2$ and $\xi>0.66$ for $N=6,\left|T_{N}\right|$ is constant. In this zone, $\left|T_{N}\right|$ strongly decreases with $N$ according to the exponential function $\left|T_{N}\right|=\left|T_{1}\right|^{N}$, as proposed by Pyrak-Nolte et al. (1990a).

4. For smaller $\xi$ values, $\left|T_{N}\right|$ reaches the maximum value. In this area, $\left|T_{N}\right|$ decreases more slowly with increasing $N$. Moreover, in the range of $\xi$ between 0 and 0.04 (0.04 corresponds to the maximum value of $\left.\left|T_{N}\right|\right),\left|T_{N}\right|$ is approximately the same with increasing $N$.

Finally, it should be noted again that only the effects of joints on wave attenuation are taken into account in the present study, since joints cause much more wave attenuation comparing with rock material damping.
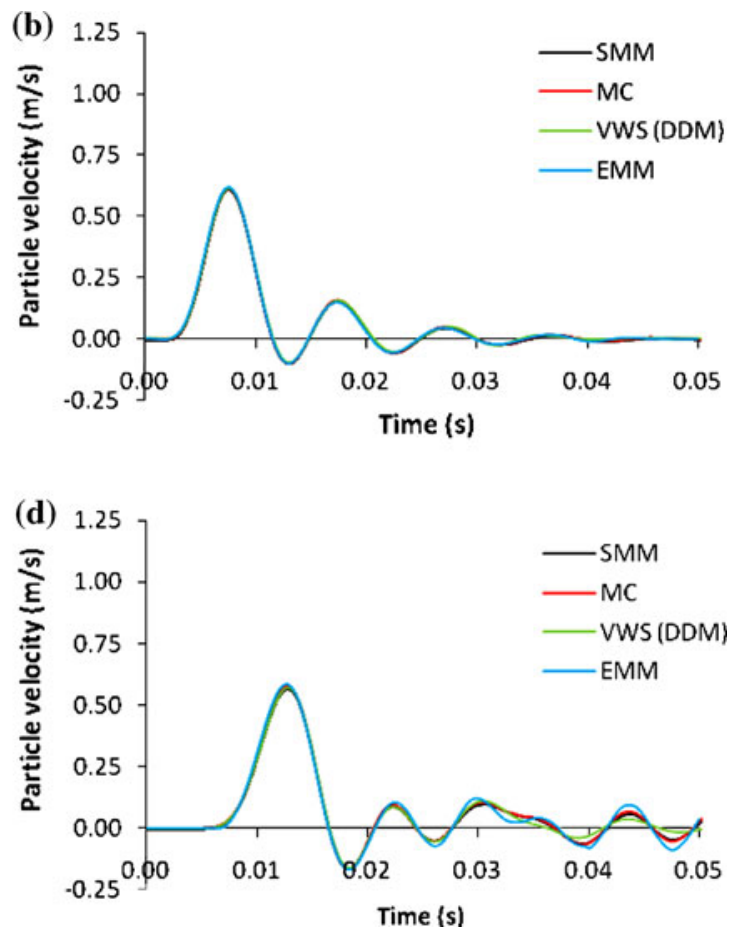

Fig. 7 Comparison of transmitted waves obtained from four methods with different joint number $N\left(K_{n}=0.36\right.$ and $\left.S=1 / 10 \lambda\right)$. a Single joint, b two joints, $\mathbf{c}$ three joints, and $\mathbf{d}$ four joints 


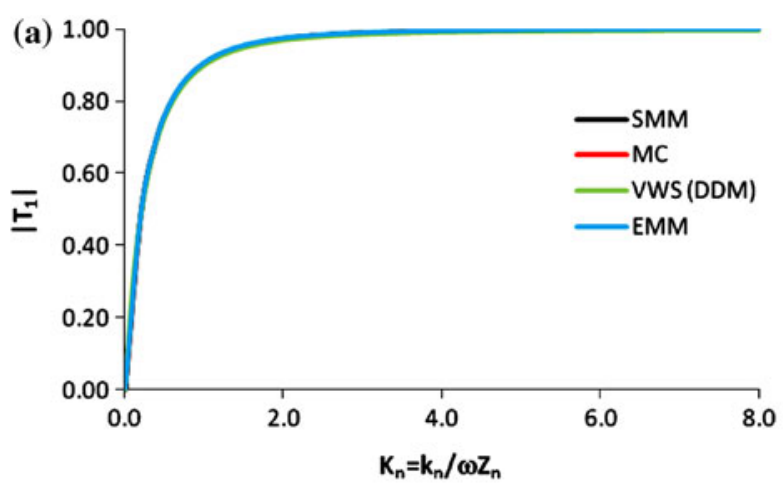

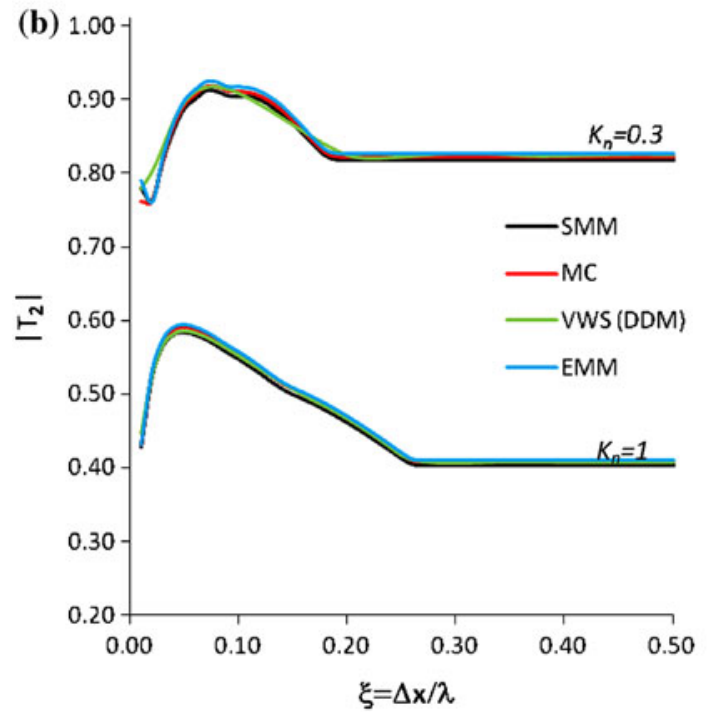

Fig. 8 Comparison of transmission coefficient obtained from four methods with different joint number $N$, normalized stiffness $K_{n}$ and joints spacing $S$. a Transmission coefficient for a single joint versus $K_{n}$, b Transmission coefficient for two parallel joints versus the non-

In fact, if the stiffness of the joint decreases, the amplitude of the transmitted wave decreases. If the frequency increases, the amplitude of the transmitted wave decreases. The reduction of the amplitude of the transmitted wave increases with the increase in the number of joints because at each joint some energy of the wave is reflected.

\subsection{Discussion}

The MC, SMM and VWS methods use the displacement discontinuity method for representing the joint as a discontinuity in displacements, while EMM adopts a different approach to model the rock mass.

$\mathrm{MC}$ is widely used in solving one-dimensional wave propagation problems and also helps to explain the boundary and initial problems that must be prescribed in such cases. The method works directly in the time domain. Therefore, there is no additional step of FFT and IFFT.

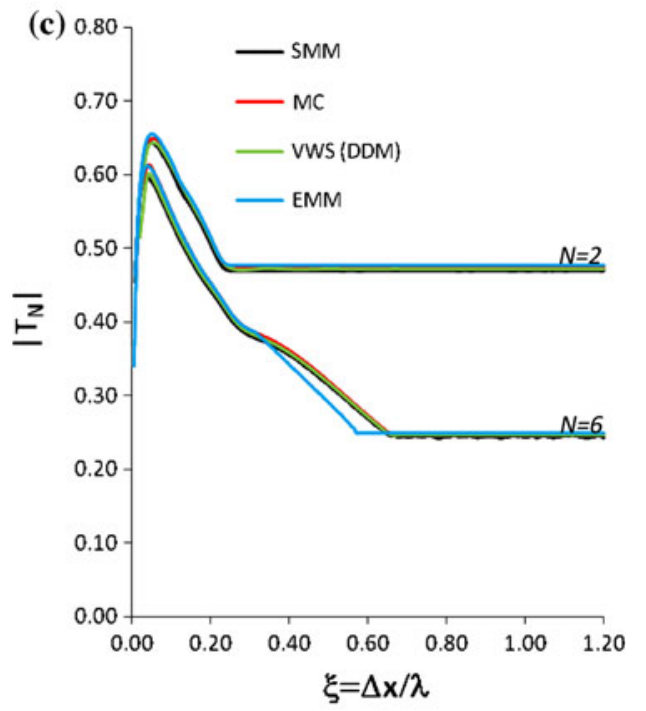

dimensional joint spacing $\xi$, and $\mathbf{c}$ Transmission coefficient for $N$ parallel joints versus the non-dimensional joint spacing $\xi$ with a $K_{n}=0.36$

With MC, wave interaction with different joint behavior can be studied, e.g., joints with nonlinear behavior (the static BB model, Zhao J et al. 2006) and joints with Coulomb slip behavior (Zhao XB et al. 2006a). MC can also be used to study wave propagation across a large number of joints and joints with different spacing. However, MC cannot be used in the frequency domain. The method uses differential equations to calculate the dynamic response and therefore it is affected by inherent computation errors. With smaller time interval, the results will be more accurate, but the computation efficiency will be lower. In addition, it is difficult for MC to study obliquely incident wave propagation across jointed rock masses.

With SMM, all multiple wave reflections between joints are taken into account. This method works in the frequency domain. Transmission and reflection coefficients for a single joint or for many joints can be derived analytically. With SMM, we can derive the analytical expressions available in literature (e.g., Pyrak-Nolte et al. 1990a). With 
this method, material damping can be considered, which in previous analyses was set equal to zero, to better evaluate the influence of joints on wave propagation. SMM can also allow the study of various joint conditions (e.g., dry or filled joints) and to treat obliquely incident waves across a joint set. Additionally, wave propagation across a large number of joints in a rock mass and joints with different spacing can be studied. However, to study wave propagation in time domain, FFT and IFFT are used to transform harmonic wave to transient wave. Thus, there will be additional computation time, and the time interval will result in computational errors. For an obliquely incident wave, some difficulties can be found in comparing the transmitted wave computed by using SMM and that measured in experiments or obtained by numerical modeling. These difficulties are due to the limited transversal size of the specimen or of the numerical model, which results in a limited number of transmitted waves from multiple wave reflections between joints.

VWS is a newly introduced concept that represents the influence of joints on wave propagation. VWS exists at each joint position and produces new waves ( 2 in opposite directions) each time an incident wave propagates across the VWS. With the transmission and reflection coefficients obtained for one single joint in the frequency domain, wave propagation across one joint set can be studied, where multiple wave reflections are considered. Material damping can also be taken into account. The method can be extended to study obliquely incident waves across one joint set with different joint spacing. In addition, with VWS, different arriving transmitted waves can be separated, and the analytical results can be compared with experiments, numerical modeling and in situ tests, especially for obliquely incident waves. However, in the time domain, FFT and IFFT should be used to transform harmonic wave to transient wave. Thus, there will be additional computation time, and the time interval will result in computational errors. Besides, the times of multiple wave reflections are controlled by the time duration used in the computation. With longer time, the times of multiple wave reflections will be larger and the results will be more accurate but the computational time will be longer.

Unlike previous methods, the EMM adopts an equivalent viscoelastic medium model and the concept of VWS to solve the problem. The VWS is adopted to reflect the discreteness of rock mass and to consider the multiple wave reflections among joints. With this method, effective moduli of the jointed rock mass can be accurately obtained. It is convenient to adopt the effective moduli to quantitatively compute the wave attenuation across the jointed rock mass in engineering application. However, this method requires some additional steps for computing the input parameters $\left[E_{v}\right.$ and $\left.\eta_{v}\right]$ that are function of the incident wave frequency and the joint spacing. EMM also needs to carry out further study for some other problems, such as the obliquely incident wave propagation problems and the wave propagation across a great number of joints with different spacing.

\section{Conclusions}

We can state that the methods described in this paper can be used to simulate wave propagation across a set of rock joints, where multiple wave reflections can be taken into account. All of them have advantages and disadvantages. Depending on the problem to be solved (type of input signal or joint conditions), a specific method can be chosen and adopted.

\section{References}

Aki K, Richards PG (2002) Quantitative seismology, 2nd edn. University Science Books, California

Bedford A, Drumheller DS (1994) Introduction to elastic wave propagation. Wiley, Chichester

Cai JG, Zhao J (2000) Effects of multiple parallel fractures on apparent wave attenuation in rock masses. Int J Rock Mech Min Sci 37:661-682

Coates RT, Schoenberg M (1995) Finite-difference modeling of faults and fractures. Geophysics 60:1514-1526

Collin R (1992) Foundations for microwave engineering. McGrawHill, New York

Cook NGW (1992) Natural joint in rock: mechanical, hydraulic and seismic behaviour and properties under normal stress. Int J Rock Mech Min Sci Geomech Abstr 29:198-223

Courant R, Hilbert D (1962) Methods of mathematical physics II. Interscience, New York

Ewing WM, Jardetzky WS, Press F (1957) Elastic waves in layered media. McGraw-Hill, New York

Frazer LN (1990) Dynamic elasticity of microbedded and fractured rocks. J Geophys Res 95:4821-4831

Hudson JA (1981) Wave speeds and attenuation of elastic waves in material containing cracks. Geophys J R Astron Soc 64:133-150

Li JC, Ma GW, Zhao J (2010) An equivalent viscoelastic model for rock mass with parallel joints. J Geophys Res 115. doi: 10.1029/2008JB006241

Miller RK (1978) The effects of boundary friction on the propagation of elastic waves. Bull Seism Assoc Am 68:987-998

Mindlin RD (1960) Waves and vibrations in isotropic, elastic plates. In: Goodier JN, Hoff NJ (eds) Structural mechanics. Pergamon, New York, pp 199-232

Myer LR, Pyrak-Nolte LJ, Cook NGW (1990) Effects of single fractures on seismic wave propagation. In: Barton N, Stephansson O (eds) Rock joints. Balkema, Rotterdam, pp 467-473

Pyrak-Nolte LJ (1988) Seismic visibility of fractures. PhD thesis. University of California, Berkeley

Pyrak-Nolte LJ, Myer LR, Cook NGW (1990a) Transmission of seismic waves across single natural fractures. J Geophys Res 95:8617-8638

Pyrak-Nolte LJ, Myer LR, Cook NGW (1990b) Anisotropy in seismic velocities and amplitudes from multiple parallel fractures. J Geophys Res 95:11345-11358 
Schoenberg M (1980) Elastic wave behavior across liner slip interfaces. J Acoust Soc Am 68:1516-1521

Schoenberg M, Muir F (1989) A calculus for finely layered anisotropic media. Geophysics 54:581-589

Slawinski RA (1999) Finite-difference modeling of seismic wave propagation in fractured media. $\mathrm{PhD}$ thesis. University of Calgary, Calgary

White JE (1983) Underground sound. Elsevier, New York

Yi W, Nihei KT, Rector JW, Nakagawa S, Myer LR, Cook NGW (1997) Frequency-dependence seismic anisotropy in fractured rock. Int J Rock Mech Min Sci Geomech Abstr 34(3/4):349-360

Zhao J, Cai JG (2001) Transmission of elastic P-waves across single fractures with a nonlinear normal deformational behavior. Rock Mech Rock Eng 34:3-22
Zhao J, Zhao XB, Cai JG, Hefny AM (2006) A further study on $\mathrm{P}$-wave attenuation across parallel fractures with linear deformational behavior. Int J Rock Mech Min Sci 43:776-788

Zhao XB, Zhao J, Cai JG (2006a) P-wave transmission across fractures with nonlinear deformational behaviour. Int $\mathrm{J}$ Numer Anal Methods Geomech 30:1097-1112

Zhao XB, Zhao J, Hefny AM, Cai JG (2006b) Normal transmission of S-wave across parallel fractures with Coulomb slip behavior. J Eng Mech 132:641-650 